\title{
THE TUMOR SUPPRESSOR NDRG2 DISRUPTS THE ONCOGENICITY OF THE CYTOPLASMIC PRMT5 IN ATL LEUKEMIA
}

\author{
OBEID SHANAB ${ }^{1}$, KAZUHIRO MORISHITA $^{2}$, AHMED Y. NASSAR $^{3}$, \\ MOHAMMED N. ISMAIL ${ }^{4}$ and MOHAMMED SALAH ${ }^{1}$. \\ ${ }^{1}$ Biochemistry Dept., Fac. Vet. Med. South Valley University, Egypt. \\ ${ }^{2}$ Tumor and Cellular Biochemistry Dept., Fac. of Medicine, University of Miyazaki, Japan. \\ ${ }^{3}$ Biochemistry Dept., Fac. of Medicine. Assiut University, Egypt. \\ ${ }^{4}$ Animal Internal Med. Dept., Fac. Vet. Med. South Valley University, Egypt.
}

Received: 12 March 2017; Accepted: 28 March 2017

\begin{abstract}
Adult T-cell leukemia (ATL) is an oncogenic disease derived from the HTLV-1-infected T cells and there is no effective therapy known yet. We previously reported that down-regulation of N-myc downstream-regulated gene-2 (NDRG2) expression by DNA Methylation and genetic deletion presents one of the most common alterations in adult T-cell leukemia (ATL) and other various kinds of cancers. A stress-induced NDRG2 suppresses important signaling pathways (PI3K and NF- $\mathrm{KB}$ ) through the de-phosphorylation of PTEN and NIK as a PP2A recruiter. In this manuscript, we identified protein arginine methyltransferase 5 (PRMT5) as a NDRG2/PP2A binding partner. A NDRG2/PP2A complex down-regulated arginine methyltransferase activity of PRMT5 through de-phosphorylation of the serine and threonine residues and changing its co-localization to the nucleus of ATL cell lines increasing the histone arginine methylation; however, PRMT5 was highly phosphorylated and localized in cytoplasm in NDRG2-deficient ATL.
\end{abstract}

Key words: NDRG2, PRMT5, PP2A, ATL, Leukemia.

\section{INTRODUCTION}

Adult T-cell leukaemia-lymphoma (ATLL) is a malignant disease caused by the oncogenic retrovirus Human T-cell leukemia virus type 1 (HTLV-1) which aggressively infect CD4+ T-cells. The manifestations of this disease appear after a long clinical latency period up to 3 decades. The Genetic, epigenetic cellular changes and the molecular mechanism of leukemogenesis that occur in HTLV-1-infected cells, which contribute to the disease development, is not completely understood. ATLL is develops in 6\% during the lifetime of an infected individual (Hinuma et al., 1981; Proietti et al., 2005; Yoshida et al., 1984). N-myc downstream-regulated gene 2 (NDRG2) was identified as a novel PTEN- associated protein that recruits protein Phosphatase 2A (PP2A) to modify the PTEN phosphorylation at the Ser380, Thr382 and Thr383 residues in its C-terminal domain and the NIK-Thr559 modification (Ichikawa et al., 2015; Nakahata et al., 2014).

Corresponding author: Dr. OBEID SHANAB

E-mail address: shanab1984@gmail.com

Present address: Biochemistry Department, Faculty of Vet. Med. South Valley University, Egypt.
The expression of the N-myc downstream-regulated gene 2 (NDRG2) was significantly down-regulated in ATLL through the DNA Methylation and genetic deletion which followed by its inactivation which is reported in many types of cancers. N-myc downstream-regulated gene 2 (NDRG2) has a crucial role in suppression of the phosphorylation of many pivotal signalling molecules in the most important signalling pathways through recruitment of PP2Ac which resulting in their dysregulation. Protein arginine methylation, catalyzed by members of the protein arginine methyltransferase (PRMT) family, which existed in the nucleus and cytoplasm (Pahlich et al., 2006). According to the methylation products, PRMTs are classified into three types (Baldwin et al., 2015). Type II PRMT that generates symmetric dimethyl arginine (SDMA) modification, PRMT5 is involved in tumorigenesis via both epigenetic silencing and organelle biogenesis (Karkhanis et al., 2011).

In the mammalian cells, PRMT5 localizes to both the cytoplasm and the nucleus and it methylates multiple histone and non-histone proteins (Bedford and Clarke, 2009). In the cytoplasm, PRMT5 forms a 20S protein arginine complex called the methylosome, consisting of PRMT5, WD repeat protein (MEP50), 
spliceosomal snRNP Sm proteins and pICln to function as a master regulator of splicing (Meister $e t$ al., 2001). PRMT5 in the cytoplasm is required for proliferation of prostate epithelial cells, while PRMT5 in the nucleus in function with the androgen receptor to drive prostate epithelial cell differentiation and function (Gao and Wang, 2012; Gu et al., 2012). Translocation of PRMT5 from the nucleus to the cytoplasm is associated with prostate tumorigenesis indicating that the Cytoplasmic PRMT5 is required for the growth of prostate cancer ( $\mathrm{Gu}$ et al., 2012). PRMT5 over-expression was found in different types of cancer, and the PRMT5 was considered as a significant target for cancer therapy (Ibrahim et al., 2014; Powers et al., 2011).
In this study, we identified new molecular mechanisms of targeting the leukemogenesis ATL, suggesting that the Cytoplasmic PRMT5 has a crucial role in the proliferation and growth of the ATL cell lines, while the nuclear PRMT5 may suppressing the growth of ATL.

\section{MATERIALS AND METHODS}

\section{Reagents}

Cell proliferation/cell toxicity Cell Counting Kit-8 was purchased from DOJINDO (Kumamoto, Japan). Most of the antibodies we used in this experiment were purchased from the companies listed in Table $\mathbf{1}$.

\begin{tabular}{|c|c|c|c|c|}
\hline Antibody against. & Manufacturer. & Catalog no. & Clone no. & $\begin{array}{l}\text { Dilution } \\
\text { factor. }\end{array}$ \\
\hline PRMT5 & SANTA CRUZ & Sc-22132 & $\mathrm{C}-20$ & $1 / 1000$ \\
\hline NDRG2 & SANTA CRUZ & Sc-19468 & E20 & $1 / 1000$ \\
\hline $\mathrm{I} \kappa \mathrm{B} \alpha$ & SANTA CRUZ & Sc-371 & $\mathrm{C}-21$ & $1 / 1000$ \\
\hline NEMO $($ IKK $\gamma)$ & SANTA CRUZ & Sc-8330 & FL-419 & $1 / 1000$ \\
\hline Histone H1 & SANTA CRUZ & Sc-8030 & $\mathrm{AE}-4$ & $1 / 1000$ \\
\hline NF-kB P52 & SANTA CRUZ & Sc-7386 & $\mathrm{C} 5$ & $1 / 1000$ \\
\hline AKT & Cell signaling & \#9272 & & $1 / 1000$ \\
\hline P-AKT (Ser473) & Cell signaling & $\# 4060$ & D9E & $1 / 1000$ \\
\hline PTEN & Cell signaling & \#9559 & 138G6 & $1 / 1000$ \\
\hline P-PTEN (Ser380/Thr382/Thr383) & Cell signaling & \#9554 & & $1 / 1000$ \\
\hline ERK1/2 & Cell signaling & $\# 4695$ & $137 \mathrm{~F} 5$ & $1 / 1000$ \\
\hline P-ERK1/2(T202-Y204) & Cell signaling & \#4094 & $\mathrm{D} 13.14 .4 \mathrm{E}$ & $1 / 1000$ \\
\hline Caspase 3 & Cell signaling & $\# 9665$ & $8 \mathrm{G} 10$ & $1 / 1000$ \\
\hline Cleaved-Caspase3 (Asp175) & Cell signaling & \#9661 & & $1 / 1000$ \\
\hline PP2Ac subunit & Cell signaling & $\# 2259$ & $52 \mathrm{~F} 8$ & $1 / 1000$ \\
\hline P-Iк B $\alpha(\operatorname{Ser} 32 / 36)(5 \mathrm{~A} 5)$ & Cell signaling & \#9246 & $5 \mathrm{~A} 5$ & $1 / 1000$ \\
\hline Myc-Tag & Cell signaling & $\# 2276$ & 9B11 & $1 / 1000$ \\
\hline$\beta$-actin & Sigma-Aldrich & A5441 & AC- 15 & $1 / 1000$ \\
\hline Flag & Sigma-Aldrich & F3165 & M2 & $1 / 1000$ \\
\hline HA & Roche & 11867423001 & $3 \mathrm{~F} 10$ & $1 / 1000$ \\
\hline P-Tyrosine & Millpore & $05-321$ & $4 \mathrm{G} 10$ & $1 / 1000$ \\
\hline P-Threonine & QIAGEN & 37420 & Q7 & $1 / 1000$ \\
\hline P-Serine & QIAGEN & 37430 & Q5 & $1 / 1000$ \\
\hline H3R8 & Abcam & Ab130740 & & $1 / 1000$ \\
\hline H4R3 & Abcam & AB5823 & & $1 / 1000$ \\
\hline TAX & Kyoto University & MI73 & & $1 / 1000$ \\
\hline Alexa Fluor-488 donkey anti-goat & Molecular Probes & A11055 & & $1 / 400$ \\
\hline Alexa Fluor-647 donkey anti-mouse & Molecular Probes & A31571 & & $1 / 400$ \\
\hline Polyclonal Rabbit anti-Mouse IgG/HPR & Dako & $\mathrm{P} 0260$ & & $1 / 1000$ \\
\hline Polyclonal Swine anti-Rabbit IgG/HPR & Dako & P0399 & & $1 / 1000$ \\
\hline Polyclonal Rabbit anti-Goat IgG/HPR & Dako & P0449 & & $1 / 1000$ \\
\hline Polyclonal Rabbit anti-Rat IgG/HPR & Dako & P0450 & & $1 / 1000$ \\
\hline
\end{tabular}




\section{Plasmids}

The Flag-tagged NDRG2 expression vector (FlagNDRG2) has been described in (Nakahata et al., 2014). The full-length complementary DNA (cDNA) sequence of PRMT5 was subcloned into the EcoRI BamHI site of the p3xFlag-myc-CMV26 expression vector (Sigma-Aldrich) (Flag-PRMT5). A DNA- based short hairpin RNA (shRNA) against PRMT5 was cloned into the BamHI-EcoRI site of the RNAiReady-pSIREN-RetroQ-ZnGreen vector (Clontech) (shPRMT5). An shluc plasmid containing a shRNA against luciferase (Clontech) was used as a control. The sense and antisense shRNA sequences against PRMT5 and mutagenic primers are listed in Table 2.

\begin{tabular}{lll}
\hline Name & Sequence (5' to 3') \\
\hline shPRMT5-3 & sense & GAGGGAGTTCATTCAGGAA \\
\cline { 2 - 3 } & anti-sense & TTCCTGAATGAACTCCCTC \\
\hline shPRMT5-4 & sense & GGCCATCTATAAATGTCTG \\
\cline { 2 - 3 } & anti-sense & CAGACATTTATAGATGGCC \\
\hline
\end{tabular}

\section{Cell culture}

Jurkat and MOLT4 are HTLV-1-negative human TALL cell lines. KOB and KK1 are IL2-dependent ATL cell lines. ED and Su9T-01 and S1T are IL2independent ATLL cell lines. MT2 and HUT102 are human T-cell lines transformed by HTLV-1 infection. The human embryonic kidney cell line 293T. IL2dependent ATL cell lines were maintained in RPMI 1640 medium (Wako) supplemented with $10 \%$ fetal bovine serum and 50 JRU per $\mathrm{ml}$ recombinant human IL2 (Takeda). HTLV-1-negative cell lines, cell lines transformed with HTLV-1 and IL2-independent ATL cell lines were maintained in the same medium without IL2. The other cell lines were cultured in RPMI 1640 or Dulbecco's modified Eagle's medium (DMEM, Wako) supplemented with $10 \%$ fetal bovine serum.

\section{Establishment of stable knockdown of PRMT5 expression in ATL cell lines}

shRNA vectors were co-transfected into 293GP cells along with the envelope plasmid pVSV-G using HilyMax reagent according to the manufacturer's protocol. After $6 \mathrm{~h}$ of transfection, the medium was changed, and the cells were incubated for $48 \mathrm{~h}$ in DMEM with $10 \%$ FBS and $10 \mu \mathrm{M}$ Forskolin (SigmaAldrich). The supernatant containing retrovirus was collected by polyethylene glycol (PEG, Wako) purification. After two days of retroviral infection in ATL cell lines (HUT102 and KOB), EGFP-positive cells were sorted with a JSAN cell sorter (Bay Bioscience).

\section{Western blot}

Cells were harvested for the extraction of proteins by homogenization in NP-40 lysis buffer $(50 \mathrm{mM}$ Tris$\mathrm{HCl}, \mathrm{pH} 8.0,150 \mathrm{mM} \mathrm{NaCl}, 5 \mathrm{mM}$ EDTA, $1 \% \mathrm{NP}-$ 40) supplemented with a proteinase inhibitor cocktail (Sigma-Aldrich) and phosphatase inhibitor tablet (PhosStop, Roche). The lysate was centrifuged for 10 $\min$ at $15,000 \times \mathrm{g}$ (maximum) at $4{ }^{\circ} \mathrm{C}$, and the supernatant was then collected. Equal amounts of protein samples were loaded, separated by SDS- polyacrylamide gel electrophoresis and then transferred to a polyvinylidene difluoride membrane (PVDF, Immobilon-P, Millipore). The membranes were blocked in PBS-Tween $(0.1 \%)$ with $1 \%$ BSA or $5 \%$ nonfat dried milk and were then probed with primary antibodies diluted in PBST-BSA or $5 \%$ nonfat dried milk. The bands were detected using a Lumi-light Plus kit (Roche) and LAS-3000. Band intensities were quantified with the NIH Image $J$ software. All primary antibodies were used at a dilution of 1:1000. For subcellular fractionation, cytoplasmic and nuclear protein extracts were prepared using NE-PER Nuclear and Cytoplasmic Extraction Reagent (Thermo Fisher Scientific). The efficiency of fractionation was confirmed by western blot analysis for $\beta$-actin (cytoplasm) and histone H1 (nucleus).

\section{Immunoprecipitation}

The lysates were incubated with $1 \mu \mathrm{g}$ of the indicated antibodies or normal $\mathrm{IgG}$ with constant rotation at 4 ${ }^{\circ} \mathrm{C}$ overnight and were then incubated with Protein $\mathrm{G}$ Sepharose 4 Fast Flow (GE Healthcare, Uppsala, Sweden) for $2 \mathrm{~h}$. The immunoprecipitates were washed 3 times with PBS, and the bound proteins were denatured in SDS sample buffer. Each sample was subjected to western blot analysis.

\section{Immunofluorescence staining}

Cells were fixed with $4 \%$ paraformaldehyde for 10 min at room temperature, washed with TBS $0.1 \mathrm{M}$ glycine, treated with $0.1 \%$ NP-40 and rewashed with TBS $0.1 \mathrm{M}$ glycine. After blocking with $1 \% \mathrm{BSA}$ in TBS, the cells were incubated with primary antibodies (1:200) overnight at $4{ }^{\circ} \mathrm{C}$. The cells were then washed three times with TBST and incubated with secondary antibodies (1:400) at room temperature for $2 \mathrm{~h}$. The coverslips were washed three times with TBST and then mounted on glass slides using an antifade reagent (Invitrogen). Nuclei were counterstained with DAPI. The proteins were visualized using a confocal laser-scanning microscope (Leica Microsystems). 


\section{Cell proliferation assay}

Cells were seed into 96-well plates at a density of $5 \times 10^{3}$ cells per well and incubated for the indicated time period. Viable cells were counted by a methyl thiazolyl tetrazolium assay using a cell counting kit-8.

\section{Trypan blue assay}

Cell growth was evaluated with a Trypan blue exclusion assay. The living cells were examined by light microscopy at low magnification after Trypan blue staining. The cell viability percentage was calculated with the following formula: (\% viable cells $=[1.00-($ Number of blue cells $\div$ Number of total cells)] $\times 100)$. This counting was repeated 3 times after 2 days in sub-culture.

\section{TUNEL assay}

For the TUNEL assay, $5 \times 10^{6}$ ATL cells were fixed with $1 \%$ paraformaldehyde, and the in situ detection of cells with DNA-strand breaks was performed by the TUNEL labeling method using an ApopTag Peroxidase In Situ Apoptosis Detection Kit, which detects apoptotic cells in situ (Millipore), according to the manufacturer's instructions. Standard deviations of three independent experiments were indicated.

\section{Cell cycle assay}

Ethanol-fixed ATL cells were stained with DAPI and incubated at $37{ }^{\circ} \mathrm{C}$ for $20 \mathrm{~min}$. At least 30,000 cell events were collected and analyzed by flow cytometry (BD FACSCalibur). Cellular DNA histograms were examined for cell cycle analysis.

\section{Statistical analysis}

The data, bars and markers in the figures represent the mean \pm s.d. We used the ANOVA for multiple comparisons and to compare each of a number of treatments with a single control. Differences were considered statistically significant when the $P$ value was $<0.05$.

\section{RESULTS}

The expression and co-localization of PRMT5 in ATL cell lines.

NDRG2 was identified as a novel PTEN binding protein for recruiting $\mathrm{PP} 2 \mathrm{~A}$, resulting in regulating PTEN Phosphatase activity by PTEN C-term phosphorylation status (Nakahata et al., 2014). We confirmed later that the PRMT5 is an NDRG2 binding protein.

We examined the expression levels of PRMT5 and NDRG2 proteins in ATL-related cell lines. As it compared with two T-ALL cell lines (Jurkat and MOLT4) as a control, the protein expression levels of PRMT5 was sustained in the ATL-related cell lines, although with the loss of NDRG2 expression (Fig. 1A). It means that, the function of PRMT5 maybe differs from normal to cancer cells. Since the oncogenic function of PRMT5 was found its localization in cytoplasm of prostate cancer cells $(\mathrm{Gu}$ et al., 2012), and as mentioned before in our results that the PRMT5 protein not differ between normal and cancer cells, we next investigate the sub-cellular localization of PRMT5 by western blot analysis after separation of nucleus and cytoplasm and immunefluorescence staining to the ATL-related cells with or without forced-expression of NDRG2. In ATLrelated cells with low expression of NDRG2, most of the PRMT5 protein was localized in cytoplasm along with PTEN, AKT, and beta-actin moreover there is a high level of the phosphorylated AKT and PTEN; however, after introduction of NDRG2 expression, a part of PRMT5 was moved into nucleus. Interestingly, although the majority of NDRG2 protein was detected in the cytoplasm, PTEN and AKT were also moved to nucleus with PRMT5 and we detected lower phosphorylated levels of AKT and PTEN which still remaining in the cytoplasm (Fig, 1 B). To confirm the localization of PRMT5 in these ATL-related cell lines with or without NDRG2 expression, immunofluorescence staining of PRMT5 and NDRG2 was done to these cell lines by the specific antibody of Alexa-488-labeled PRMT5 (green) and Alexa Fluor-647-labeled NDRG2 (red), respectively. In ATL-related cells (HUT102 and $\mathrm{KOB}$ ) with low expression of NDRG2, the majority of the green-labeled PRMT5 was detected in the cytoplasm without merge with DAPI-stained nucleus (blue). On the other hand, after introduction of NDRG2, PRMT5 was detected both in the cytoplasm and the nucleus (Fig. 1C). Furthermore the histone modifications (histone arginine methylatin which is a target for PRMT5) in the cell expressing the exogenous NDRG2 were confirmed by the western blot analysis (Fig. 1D).

\section{PRMT5 in ATL-related cells loss it's activity for enhancing the cell growth after NDRG2 expression.}

To investigate whether the function of PRMT5 in ATL-related cells is dependent on the loss of NDRG2 expression, we established ATL-related cells with forced expression of NDRG2 and/or the knockdown expression vector for PRMT5 (shPRMT5-3/NDRG2 and shPRMT5-4/NDRG2 in HUT102 and KOB). After confirmation of the expression levels of NDRG2 and PRMT5 by western blot analysis (Figs $2 \mathrm{~A}$ and $2 \mathrm{C}$ ), we determined the cell growth rates (Figs 2B \&2D), where the transformat cells with high expression of NDRG2 showed reduction of cell growth with enhanced apoptosis with or without PRMT5 knockdown. The apoptosis of the transformant cells with NDRG2 expression was detected by presence of the cleaved caspase 3 as shown in (Fig 2E \&2F). Otherwise in the NDRG2 transformant (HUT102 and KOB) cells with or without PRMT5 KD most of these signals not 
significantly changed (Figure $2 \mathrm{G}$ ), in compare to the low NDRG2 expression in the parental lane in both HUT102 and KOB.

PRMT5 regulates the cell cycle and apoptosis in ATL:

The cell cycle checked by flow cytometry showing increased population of sub-G1 fraction in the NDRG2 transformant cells whatever the PRMT5 down-regulated or not (Fig 2A). Moreover the DNA staining using the TUNEL assay showing the damage of the DNA in the NDRG2 transformant cells which enhancing the apoptotic pathways (Fig 2B), also the cell viability was decrease in comparison with the NDRG2 negative cells (Fig 2C) by the trypan blue exclusion assay, suggesting that high expression of NDRG2 abrogated the effect of PRMT5 knockdown. Therefore, the cell growth of NDRG2low cell lines is mostly dependent on the expression of PRMT5 and the function of PRMT5 in NDRG2low cell lines is possible dependent on modified phosphorylation status of PRMT5 by the loss of NDRG2 expression.

PRMT5 is a novel binding protein to the NDRG2/PP2Ac complex which regulates its phosphorylation status:
To confirm the PRMT5 as a new binding protein of NDRG2, Flag-tagged NDRG2 expression vector was transfected to HUT102 or KOB/ATL-related cell lines and then this exogenous NDRG2 and the PP2Ac (SERINE and THREONINE Phosphatase) complex could specifically bind to endogenous PRMT5 in both ATL-related cell lines (Figs 3A \&3B). Then we checked the phosphorylation status of the PRMT5 in both HUT102 and KOB cell lines with or without NDRG2 expression by immunoprecipitation, interestingly we found that in the cell line with low NDRG2 expression showing highly phosphorylated PRMT5, while those with high expression of NDRG2 showing low phosphorylated PRMT5 (Figs 3C \&3D). Hence the NDRG2/PP2Ac complex is a serine and threonine Phosphatase; we noticed that also the tyrosine residue phosphorylation was decreased. To confirm whether PP2A could de-phosphorylate the PRMT5, we transfected the HEK293T cells with MYC-PP1c, PP2Ac or PP5c and FLAG-PRMT5, by immunoprecipitation by using the indicated antibodies we detected that the PP2Ac is strongly bind to the FLAG-PRMT5 than the PP1c or the PP5c which is weekly bind to the complex (Fig. 4F), suggesting that the PP2Ac is the most important Phosphatase bind to the PRMT5 and regulating its phosphorylation and moreover its oncogenic activity.

\section{Figure 1}
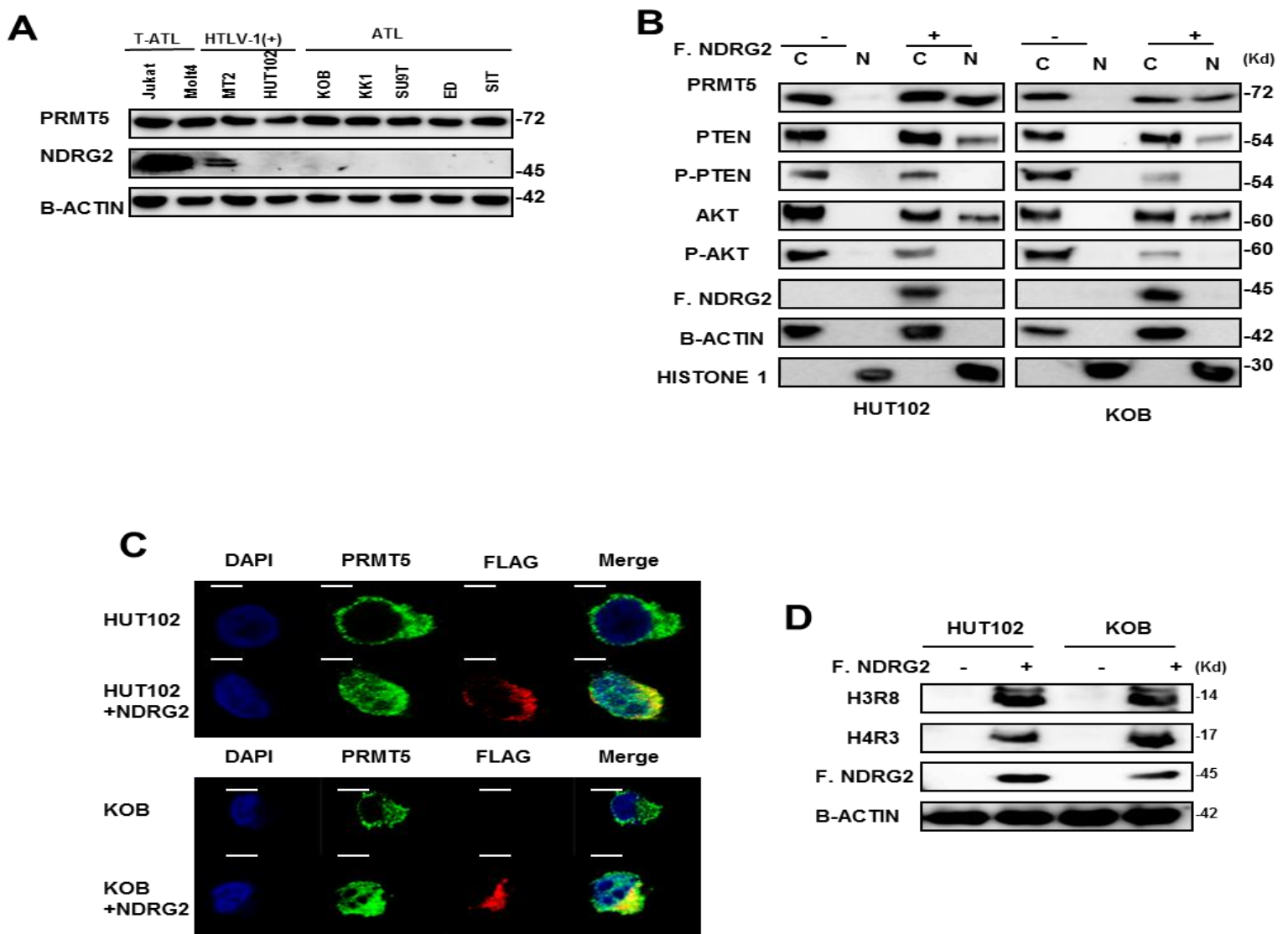
Figure 2

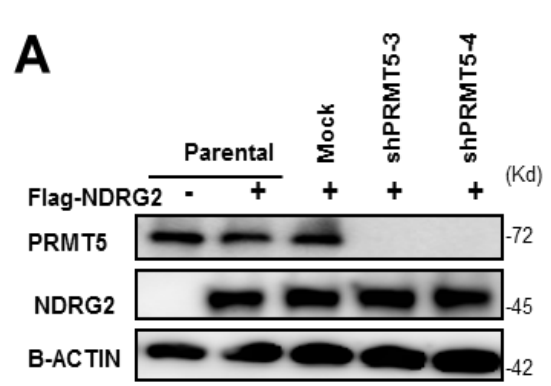

HUT102

C

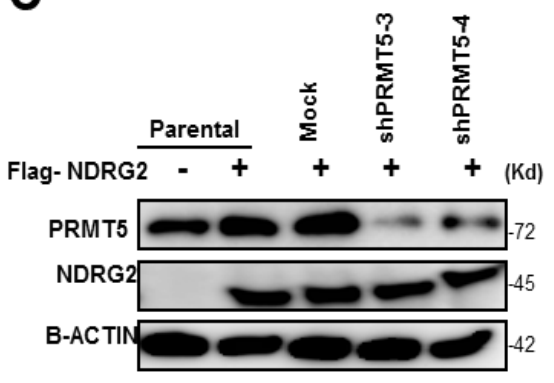

Ков
B

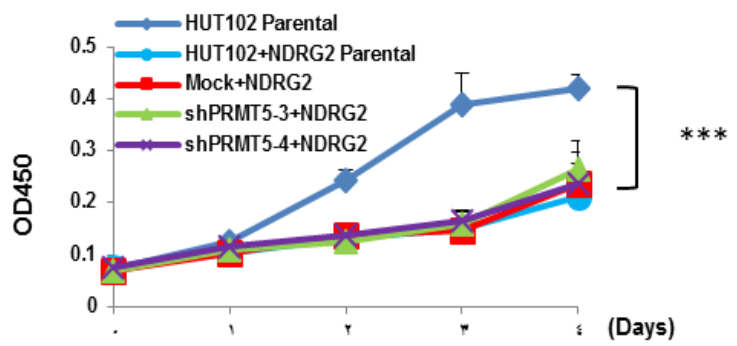

HUT102

D

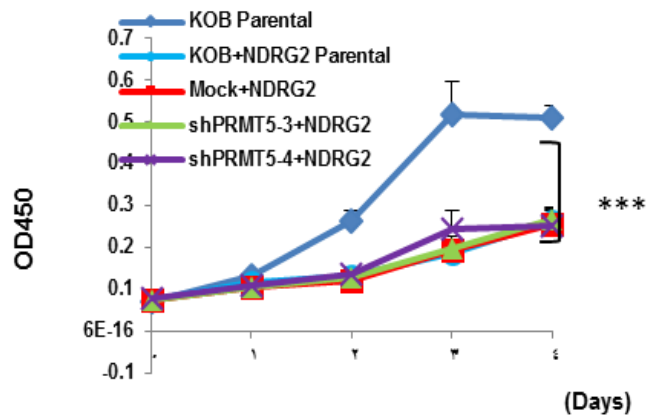

ков
E

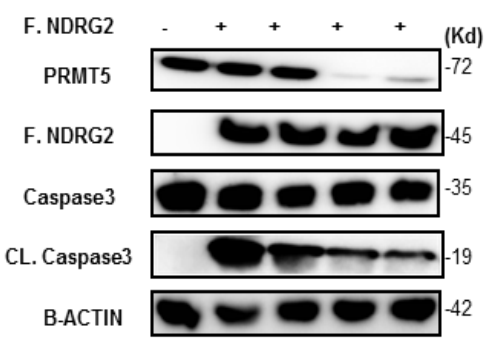

HUT102

\section{$\mathbf{F}$}

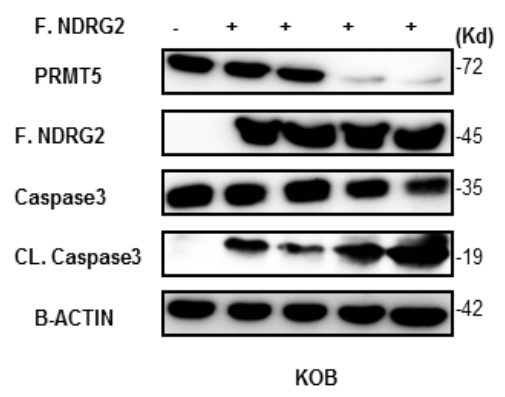

G

F. NDRG2

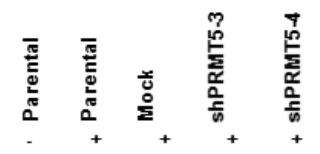

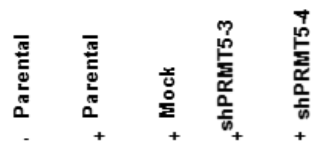

PRMT5

P-AKT

AKT

P.PTEN

PTEN

P-ERK1/2

ERK1/2

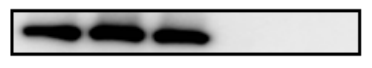

- - - -
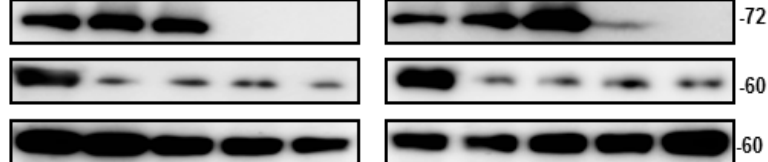

$0-60$

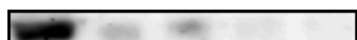

60

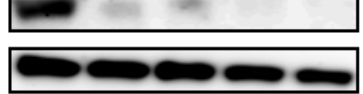

$--1-54$
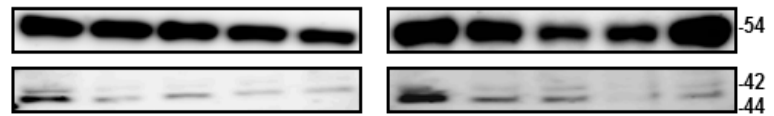

NF-KB P52

IKBa

P-IKBa

-IKBa

NEMO

TAX

F. NDRG2

B-ACTIN
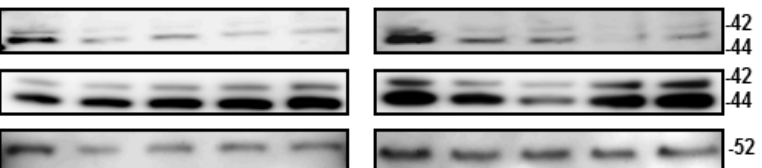

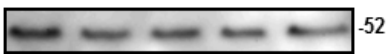
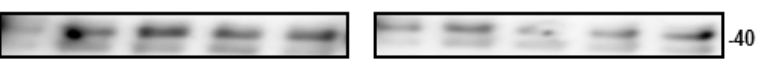

$-\cdots+\cdots$

$\because \cdots \cdots-\cdots$

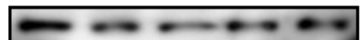

$0-\cdots+\cdots+\infty$

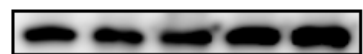

$--45$

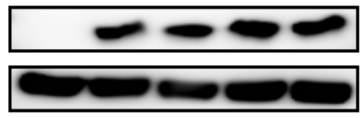

HUT102

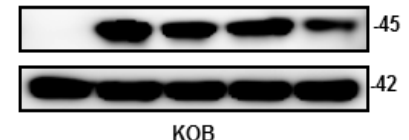


A

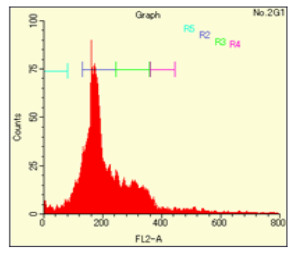

Parental

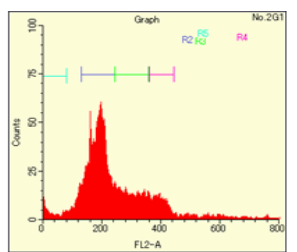

Parental

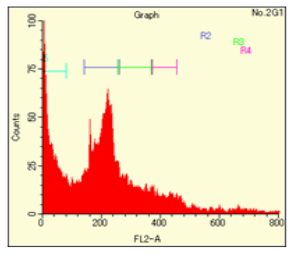

Parental +NDRG2

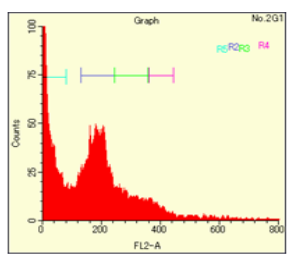

Parental +NDRG2

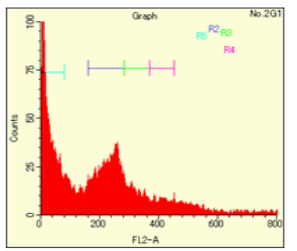

Mock +NDRG2

HUT102

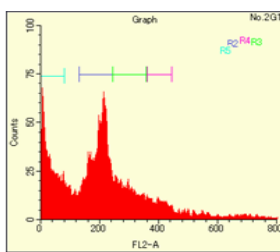

Mock +NDRG2

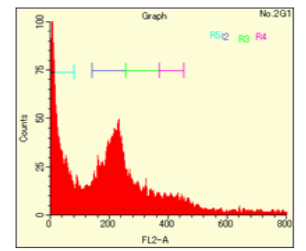

ShPRMT5-3 +NDRG2

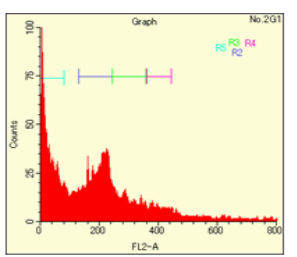

ShPRMT5-3 +NDRG2
Figure 3
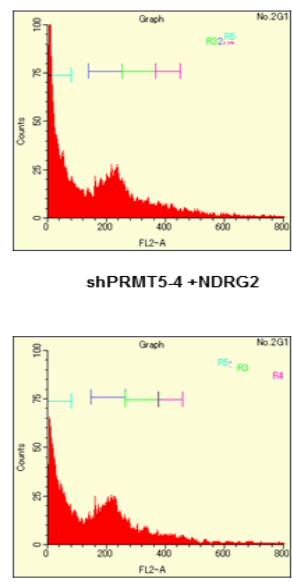

shPRMT5-4 +NDRG2

ков

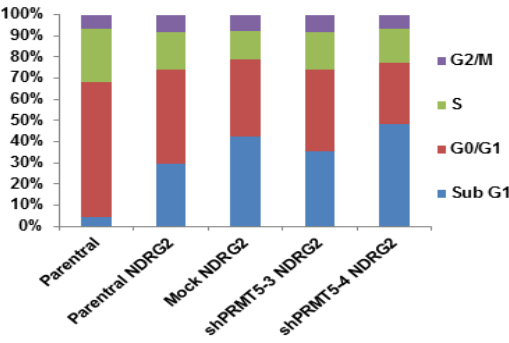

HUT102 NDRG2

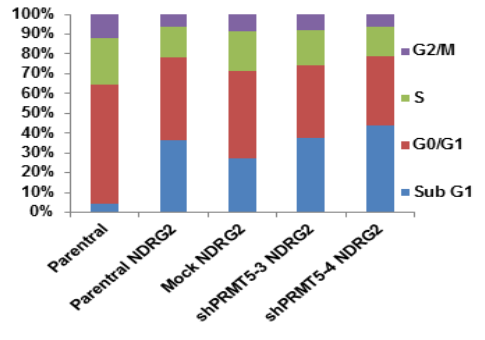

KOB NDRG2
B
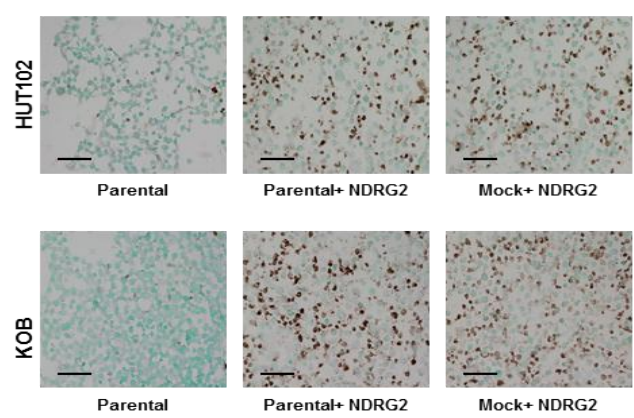

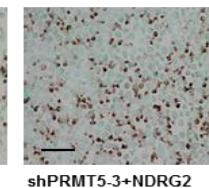

ShPRMT5-3+NDRG2

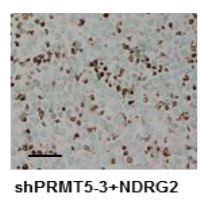

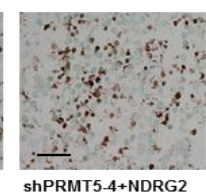

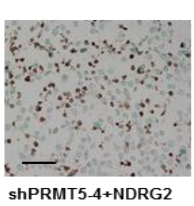

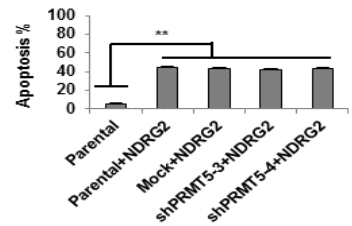

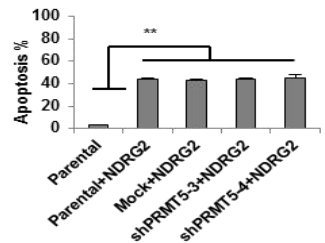

C
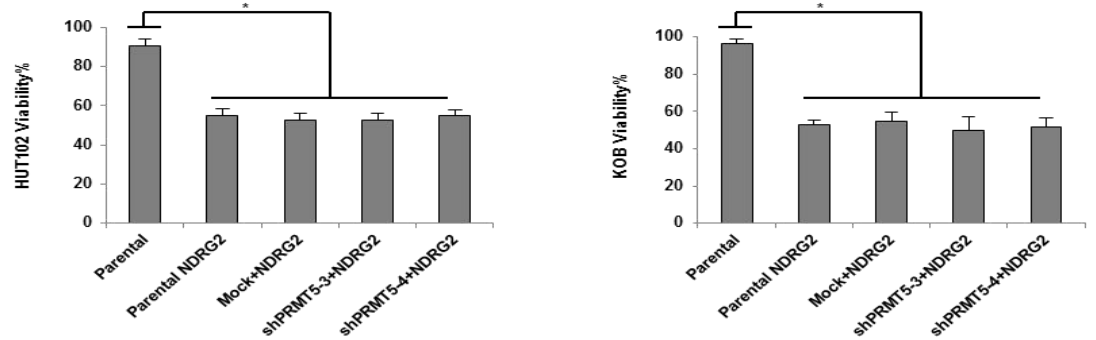


\section{Figure4}

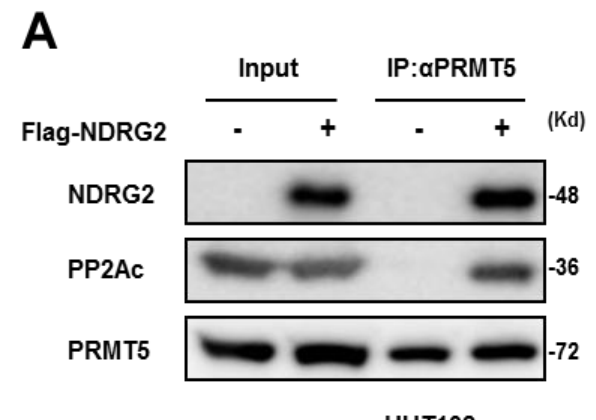

$\begin{array}{llll}\text { C F. NDRG2 } & \text { IP: PRMT5 } \\ & +(\mathrm{Kd})\end{array}$
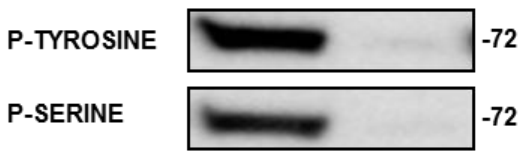

P-THREONINE

PRMT5

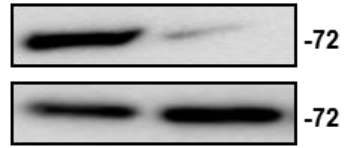

HUT102

E

FLAG PRMT5

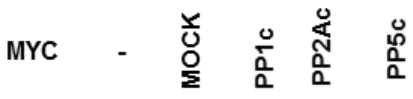
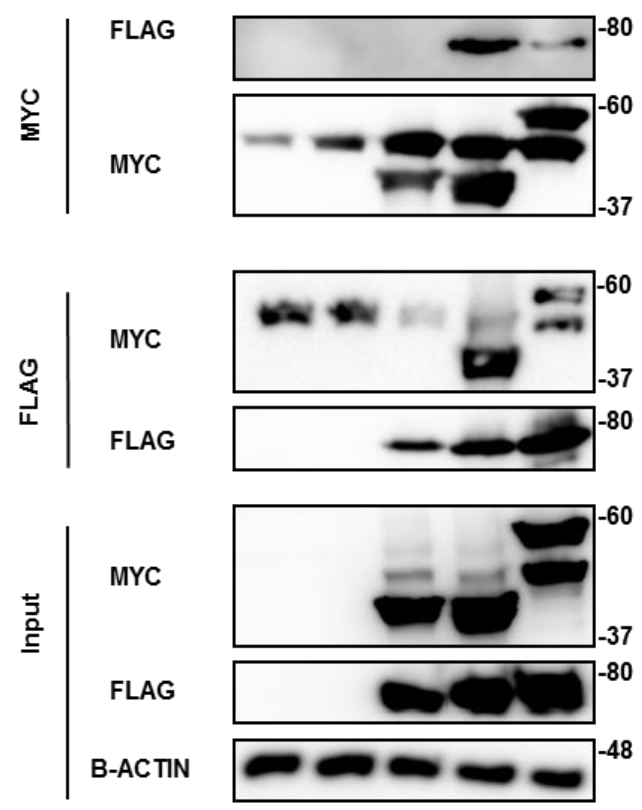

HEK 293T
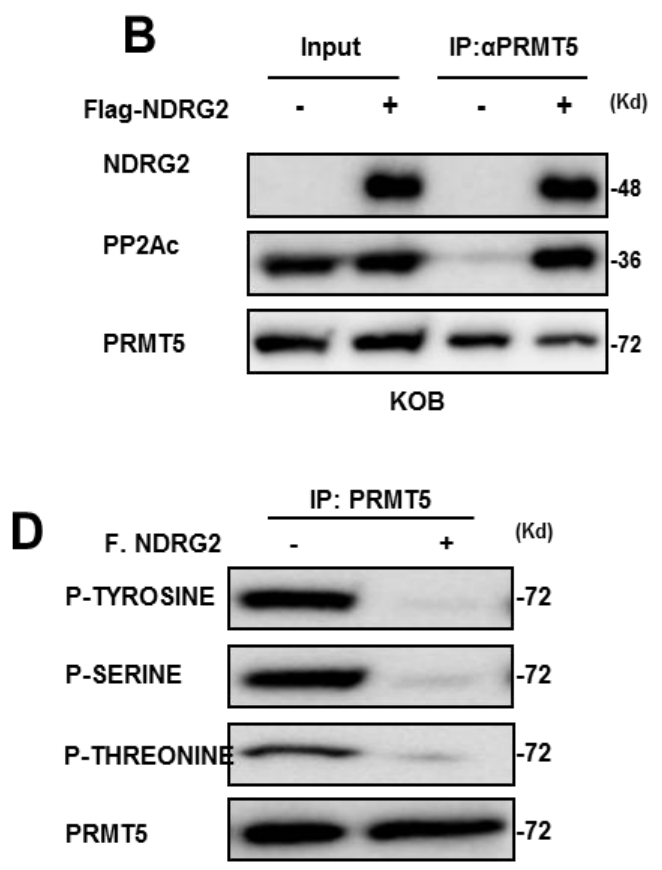

Ков 
Figures ligands.

Figure 1: The expression and co-localization of PRMT5 in ATL cell lines.

(A) Expression of PRMT5 and NDRG2 proteins were determined by immunoblot analysis using specific antibodies in 7 ATL-related cell lines and two T-ALL cell lines as indicated in the figure.

(B) After separating the nuclear and cytoplasmic fractions, the lysates of ATL-related cells were immunoblotted with anti-PRMT5, anti-NDRG2 and other important oncogenic proteins. To confirm the subcellular localization, an anti-histone $\mathrm{H} 1$ antibody was used for the nucleus and an anti- $\beta$-actin antibody for the cytoplasm.

(C) Immunofluorescence staining of HUT102 and KOB cells that were mock transfected or transfected with an NDRG2 expression vector was performed with anti-human PRMT5 antibody with Alexa Fluor488-conjugated anti-goat $\operatorname{IgG}$ antibody (green) and anti-FLAG with Alexa Fluor-647-conjugated antimouse IgG antibody (red), along with DAPI staining for the nucleus. Scale bar, $10 \mu \mathrm{m}$.

(D) The lysates of ATL-related cells were immunoblotted with anti-histone H3R8me2 or H4R3me 2 and anti-NDRG2. Anti $\beta$-actin antibody for the control.

Figure 2: PRMT5 in ATL-related cells loss it's activity for enhancing the cell growth after NDRG2 expression.

(A) And $(\boldsymbol{C})$ Immunoblot analysis of PRMT5 was performed in the NDRG2 transfected/ATL-related cell lines HUT102 or KOB cells expressing shPRMT5 and also the expression of NDRG2 was shown in ATL cell lines, $\beta$-actin as a control.

(B) And $(\boldsymbol{D})$ Cell growth curves of the NDRG2 transfected/ATL-related cell line HUT102 over four days, including parental, NDRG2 parental, mocktransfected, and shPRMT5-transfected cells. (D) The same experiment was performed in the NDRG2 transfected/ATL-related cell line KOB. The statistical analysis of the growth of the control cells versus the shPRMT5-transfected cells indicated that $\mathrm{P}<0.001$ (ANOVA).

(E) And $(\boldsymbol{F})$ Cleaved caspase 3, as an indicator for apoptosis, was identified in NDRG2 transfected/HUT102 or KOB cell lines with NDRG2 expression by an immunoblot analysis, along with PRMT5, total caspase 3 and $\beta$-actin as a control.

(G) Expression of various types of cellular proteins, such as signal transduction or cell cycle regulators, was analyzed in NDRG2 transfected/ATL-related cell line HUT102 or KOB cell lines (parental, NDRG2 parental, mock- or shPRMT5 transfected cells) by specific antibodies.

Figure 3: PRMT5 regulates the cell cycle and apoptosis in ATL.

(A) Cell cycle profiles in the NDRG2 transfected/ATL-related cell lines HUT102 or KOB cells expressing shPRMT5 and also the expression of NDRG2 were assessed by FACS analysis after DAPI staining.

(B) Cell apoptosis was evaluated by a TUNEL assay in the same cells as in $(\boldsymbol{A})$. Apoptotic cells were stained brown (left panel). The bar graph represents the percentages of apoptotic cells (right panel). Scale bar, $100 \mu \mathrm{m}$. The mean \pm s.d. is shown, $\mathrm{P}<0.001$ (ANOVA).

(C) The cell viability detected by TRYBAN BLUE exclusion test the same as in $(\boldsymbol{A})$. The data are representative of three experiments. The mean \pm s.d. is shown, $\mathrm{P}<0.001$ (ANOVA).

Figure 4: PRMT5 is a novel binding protein to the NDRG2/PP2Ac complex which regulates its phosphorylation status;

$(\boldsymbol{A})$ And (B) After Flag-tagged NDRG2 was transfected into the HUT102 and KOB /ATL-related cells and the cell lysates were precipitated by an antiPRMT5antibody, the precipitated proteins were immunoblotted with specific antibodies (antiPRMT5, anti-PP2Ac or anti-NDRG2).

(C) And (D) Co-immunoprecipitation using PRMT5 antibody with the lysates of HUT102 and KOB /ATL-related cells and blotted against the antibodies of anti-tyrosine, anti-serine and anti-threonine respectively and the PRMT5 as a control.

(E) HEK293T cells transfected with MYC-PP1c, PP2Ac or PP5c and FLAG-PRMT5, and then the interaction was checked by immunoprecipitation by using the indicated antibodies.

\section{DISCUSSION}

The expression of N-myc downstream-regulated gene 2 (NDRG2) was significantly downregulated in ATL through DNA methylation and genetic modifications (Nakahata et al., 2014). The downregulation of NDRG2 associated with tumor growth, progression and metastasis as the deletion of NDRG2 was reported in a wide variety of cancers, including pancreatic cancers, oral cancers and gastric cancers (Furuta et al., 2010; Hu et al., 2016; Yao et al., 2008). NDRG2, is a stress-responsive gene that have a role in suppressing the phosphorylation of many important signaling molecules in several signaling pathways through the recruitment of the protein 
phosphatase PP2A, which results in dephosphorylation and the maintenance of cellular homeostasis after the over-activation of stress response factors (Nakahata et al., 2014). NDRG2 is a novel PTEN-binding protein that activates PTEN phosphatase activity by recruiting PP2A, which dephosphorylates PTEN at Ser380, Thr382 and Thr383 (STT) in its C-terminal domain (C-term). In most ATL cells and oral cancers, the expression of wild-type PTEN is sustained with low phosphatase activity by maintaining the highly phosphorylated status of the PTEN C-term (STT), resulting in the constitutive activation of the PI3K/AKT signaling pathway, Moreover, we identified that NF- $\kappa \mathrm{B}$ inducible kinase (NIK) is a novel binding partner of NDRG2 (Ichikawa et al., 2015; Nakahata et al., 2014). Since NDRG2, as a tumor suppressor, is downregulated or undetectable in many human cancers, identifying the molecular mechanism of NDRG2 is important to the development of therapeutic interventions. Down-regulation of NDRG2 has been identified in some types of solid tumors, including $80.4 \%$ of OSCC and $73.9 \%$ of pancreatic cancers (Furuta et al., 2010; Yamamura et $a l ., 2013)$. Here in ATL the NDRG2 not expressed and we identified the PRMT5 as a new binding partner of the NDRG2/PP2Ac complex.

The methyltransferase activity of PRMT5 is disrupted by its phosphorylation at Y297, Y304, and Y307 by a mutated JAK kinase (V617F) (Liu et al., 2011). In embryonic stem cells, cytoplasmic PRMT5 has a role in maintenance of pleuripotency (Tee et al., 2010). The HTLV-1-transformed T cells display constitutive activation of STAT3 and STAT5 (Migone et al., 1995), so the activation of STAT signaling pathway might be involved to the cytoplasmic PRMT5 function in ATL. The tyrosine phosphorylation of PRMT5 by JAK2-V617K inhibits its arginine methyltransferase activity on histone proteins.

Among the known substrates of PRMT5 are histones H4R3 and H3R8. It has been shown that symmetric dimethylation (me2) of H4R3 and H3R8 lead to transcription repression, whereas that of H3R8 has also been associated with transcriptional activation (Fabbrizio et al., 2002; Richard et al., 2005).

In this study the arginine methylation status of histones $\mathrm{H} 3$ and $\mathrm{H} 4$ was determined in ATL-related cell lines using a specific antibody for H3R8me2 and H4R3me2 before and after NDRG2 transfection. The arginine methylation of histones $\mathrm{H} 3$ and $\mathrm{H} 4$ could not be detected in the ATL-related cells (Figure 1D); however, after the over-expression of NDRG2 in the ATL-related cell lines HUT102 or KOB, the PRMT5 translocated to the nucleus, and enhanced arginine methylation of histone $\mathrm{H} 3$ (H3R8me2) and H4 (H4R3me2) (Figure 1D). In our study the relocalized
PRMT5 in the nucleus might modulate the arginine methylation of histone $\mathrm{H} 3$ ariginine 8 (H3R8) and of histone $\mathrm{H} 4$ arginine 3 (H4R3) through nuclear transport signal by unknown modification of PRMT5. Therefore, the cytoplasmic PRMT5 accelerated their cell growth of ATL-related cells and the oncogenic function of PRMT5 might be dependent on the loss of NDRG2 expression in ATL-related cells. Here we confirmed that the PRMT5 function is related to the sub-cellular localization, hence the Cytoplasmic PRMT5 work as oncogenic factor with a high phosphorylation status, while that in the nucleus may be work as a tumor suppressor due to the histone modification which in turn down-regulates the genes which responsible for the cell growth after losing its phosphorylation by the NDRG2/PP2Ac complex. Overall as a part of the dephosphorylated PRMT5 translocated to the nucleus, resulting in enhanced histone arginine methylation.

Interestingly, STAT signaling pathway with high metastatic potential is inhibited by NDRG2 expression in several types of tumors (Wang et al., 2014). The loss of NDRG2 expression might enhance the cytoplasmic PRMT5 expression through activation of the STAT signaling pathway. Moreover, the cytoplasmic PRMT5 expression is correlated with poor prognosis.

So far the activity of PRMT5 in ATL cell lines depends on its Cytoplasmic co-localization and the phosphorylation status in which enhances the cell growth and the metastasis through the activation of some oncogenic signaling pathways and inhibition of histone arginine methylation, while the forced transfection of NDRG2 to the ATL cell lines recruited the protein Phosphatase PP2Ac to the PRMT5 resulting in its de-phosphorylation, moreover nuclear localization and histone arginine modifications, which inactivates the genes that responsible for the cell growth.

In conclusion, our in vitro data indicated that the oncogenic activity of PRMT5 was depends on its cytoplasmic localization and the phosphorylation status which is essential for ATL development. Moreover it is a critical activator for the regulatory pathways affecting cell growth, survival, migration and tumor cells activity. Furthermore, the PRMT5 can bind with the NDRG2/PP2Ac complex which responsible for its de-phosphorylation and changing its co-localization from the cytoplasm to the nucleus which in turn modify the histone $\mathrm{H} 3$ and $\mathrm{H} 4$ through its arginine methylation and lowering the growth of ATL.

So cytoplasmic PRMT5 is considered as a novel target gene in the ATL, as targeting of PRMT5 with a drugs that regulates its phosphorylation status maybe used as a novel therapeutic pattern in ATL leukemia. 


\section{REFERENCES}

Baldwin, R.M.; Haghandish, N.; Daneshmand, M.; Amin, S.; Paris, G.; Falls, T.J.; Bell, J.C.; Islam, S. and Cote, J. (2015): Protein arginine methyltransferase 7 promotes breast cancer cell invasion through the induction of MMP9 expression. Oncotarget 6, 3013-3032.

Bedford, M.T. and Clarke, S.G. (2009): Protein arginine methylation in mammals: who, what, and why. Molecular cell 33, 1-13.

Fabbrizio, E.; El Messaoudi, S.; Polanowska, J.; Paul, C.; Cook, J.R.; Lee, J.H.; Negre, V.; Rousset, M.; Pestka, S.; Le Cam, A. and Sardet, C. (2002): Negative regulation of transcription by the type II arginine methyltransferase PRMT5. EMBO reports 3, 641-645.

Furuta, H.; Kondo, Y.; Nakahata, S.; Hamasaki, M.; Sakoda, S. and Morishita, K. (2010): NDRG2 is a candidate tumor-suppressor for oral squamous-cell carcinoma. Biochemical and biophysical research communications 391, 1785-1791.

Gao, S. and Wang, Z. (2012): Subcellular localization of p44/WDR77 determines proliferation and differentiation of prostate epithelial cells. PloS one 7, e 49173.

Gu, Z.; Li, Y.; Lee, P.; Liu, T.; Wan, C. and Wang, Z. (2012): Protein arginine methyltransferase 5 functions in opposite ways in the cytoplasm and nucleus of prostate cancer cells. PloS one 7, e44033.

Hinuma, Y.; Nagata, K.; Hanaoka, M.; Nakai, M.; Matsumoto, T.; Kinoshita, K.I.; Shirakawa, S. and Miyoshi, I. (1981): Adult T-cell leukemia: antigen in an ATL cell line and detection of antibodies to the antigen in human sera. Proceedings of the National Academy of Sciences of the United States of America 78, 6476-6480.

Hu, W.; Fan, C.; Jiang, P.; Ma, Z.; Yan, X.; Di, S.; Jiang, S.; Li, T.; Cheng, $Y$. and Yang, $Y$. (2016): Emerging role of N-myc downstreamregulated gene 2 (NDRG2) in cancer. Oncotarget 7, 209-223.

Ibrahim, R.; Matsubara, D.; Osman, W.; Morikawa, T.; Goto, A.; Morita, S.; Ishikawa, S.; Aburatani, H.; Takai, D. and Nakajima, J. et al. (2014): Expression of PRMT5 in lung adenocarcinoma and its significance in epithelial-mesenchymal transition. Human pathology 45, 1397-1405.

Ichikawa, T.; Nakahata, S.; Fujii, M.; Iha, H. and Morishita, K. (2015): Loss of NDRG2 enhanced activation of the NF-kappaB pathway by PTEN and NIK phosphorylation for ATL and other cancer development. Scientific reports 5,12841 .
Karkhanis, V.; Hu, Y.J.; Baiocchi, R.A.; Imbalzano, A.N. and Sif, S. (2011): Versatility of PRMT5induced methylation in growth control and development. Trends in biochemical sciences 36, 633-641.

Liu, F.; Zhao, X.; Perna, F.; Wang, L.; Koppikar, P.; Abdel-Wahab, O.; Harr, M.W.; Levine, R.L.; $X u, \quad H$. and Tefferi, A. et al. (2011): JAK2V617F-mediated phosphorylation of PRMT5 downregulates its methyltransferase activity and promotes myeloproliferation. Cancer cell 19, 283-294.

Meister, G.; Eggert, C.; Buhler, D.; Brahms, H.; Kambach, C. and Fischer, U. (2001): Methylation of $\mathrm{Sm}$ proteins by a complex containing PRMT5 and the putative U snRNP assembly factor pICln. Current biology : CB 11, 1990-1994.

Migone, T.S.; Lin, J.X.; Cereseto, A.; Mulloy, J.C.; O'Shea, J.J.; Franchini, G. and Leonard, W.J. (1995): Constitutively activated Jak-STAT pathway in T cells transformed with HTLV-I. Science 269, 79-81.

Nakahata, S.; Ichikawa, T.; Maneesaay, P.; Saito, Y.; Nagai, K.; Tamura, T.; Manachai, N.; Yamakawa, N.; Hamasaki, M. and Kitabayashi, I. et al. (2014): Loss of NDRG2 expression activates PI3K-AKT signalling via PTEN phosphorylation in ATLL and other cancers. Nature communications 5, 3393.

Pahlich, S.; Zakaryan, R.P. and Gehring, H. (2006): Protein arginine methylation: Cellular functions and methods of analysis. Biochimica et biophysica acta $1764,1890-1903$.

Powers, M.A.; Fay, M.M.; Factor, R.E.; Welm, A.L. and Ullman, K.S. (2011): Protein arginine methyltransferase 5 accelerates tumor growth by arginine methylation of the tumor suppressor programmed cell death 4. Cancer research 71, 5579-5587.

Proietti, F.A.; Carneiro-Proietti, A.B.; CatalanSoares, B.C. and Murphy, E.L. (2005): Global epidemiology of HTLV-I infection and associated diseases. Oncogene 24, 6058-6068.

Richard, S.; Morel, M. and Cleroux, P. (2005): Arginine methylation regulates IL-2 gene expression: a role for protein arginine methyltransferase 5 (PRMT5). The Biochemical journal 388, 379-386.

Tee, W.W.; Pardo, M.; Theunissen, T.W.; Yu, L.; Choudhary, J.S.; Hajkova, P. and Surani, M.A. (2010): Prmt5 is essential for early mouse development and acts in the cytoplasm to maintain ES cell pluripotency. Genes \& development 24, 2772-2777.

Wang, J.; Yin, D.; Xie, C.; Zheng, T.; Liang, Y.; Hong, X.; Lu, Z.; Song, X.; Song, R. and Yang, H. et al. (2014): The iron chelator Dp44mT inhibits hepatocellular carcinoma metastasis via N-Myc downstream-regulated gene 2 
(NDRG2)/gp130/STAT3 pathway. Oncotarget $5,8478-8491$.

Yamamura, A.; Miura, K.; Karasawa, H.; Morishita, K.; Abe, K.; Mizuguchi, Y.; Saiki, Y.; Fukushige, S.; Kaneko, N. and Sase, T. et al. (2013): Suppressed expression of NDRG2 correlates with poor prognosis in pancreatic cancer. Biochemical and biophysical research communications 441, 102-107.

Yao, L.; Zhang, J. and Liu, X. (2008): NDRG2: a

Myc-repressed gene involved in cancer and cell stress. Acta biochimica et biophysica Sinica 40, 625-635.

Yoshida, M.; Seiki, M.; Yamaguchi, K. and Takatsuki, $K$. (1984): Monoclonal integration of human $\mathrm{T}$-cell leukemia provirus in all primary tumors of adult T-cell leukemia suggests causative role of human $\mathrm{T}$-cell leukemia virus in the disease. Proceedings of the National Academy of Sciences of the United States of America $81,2534-2537$

\section{ال ان دى ار جى الثانى المثبط للأورام يحدث خلاً فى قدرة ال بع ار ام تى الخامس المتواجد فى السيتوبلازم على احداث التورم فى مرض الابيضاض التائى الخلايا.}

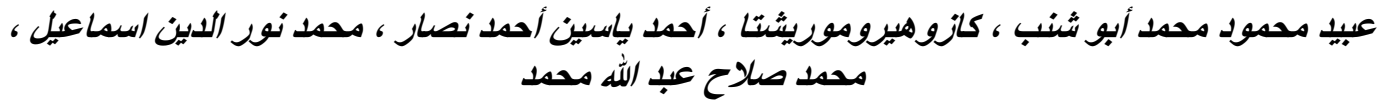

Email: shanab1984@gmail.com

Assiut University web-site: www.aun.edu.eg

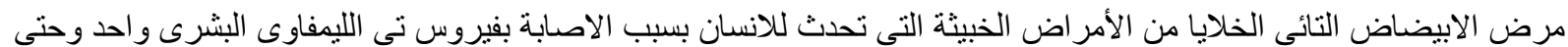

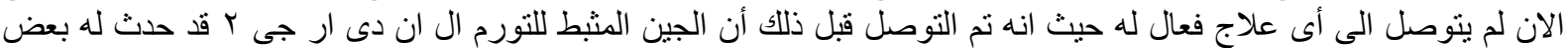

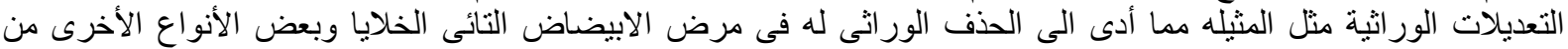

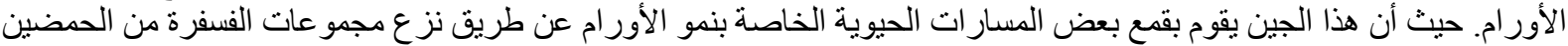

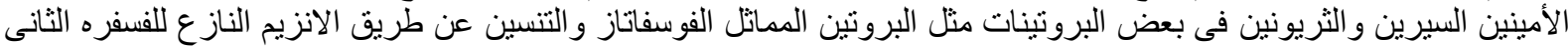

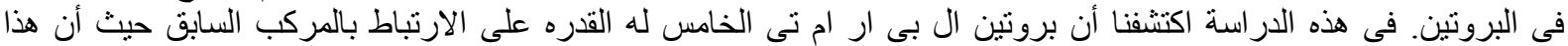

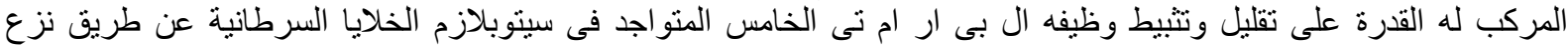

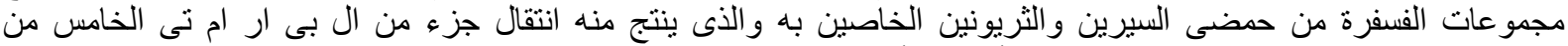

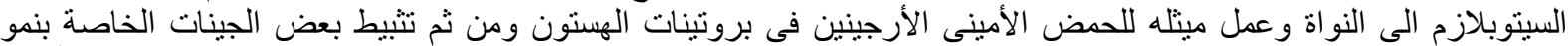

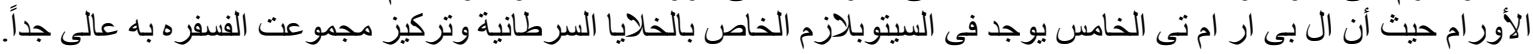

